\title{
Some Remarks on Real Numbers Induced by First-Order Spectra
}

\author{
Sune Kristian Jakobsen and Jakob Grue Simonsen
}

\begin{abstract}
The spectrum of a first-order sentence is the set of natural numbers occurring as the cardinalities of finite models of the sentence. In a recent survey, Durand et al. introduce a new class of real numbers, the spectral reals, induced by spectra and pose two open problems associated to this class. In the present note, we answer these open problems as well as other open problems from an earlier, unpublished version of the survey.

Specifically, we prove that (i) every algebraic real is spectral, (ii) every automatic real is spectral, (iii) the subword density of a spectral real is either 0 or 1, and both may occur, and (iv) every right-computable real number between 0 and 1 occurs as the subword entropy of a spectral real.

In addition, Durand et al. note that the set of spectral reals is not closed under addition or multiplication. We extend this result by showing that the class of spectral reals is not closed under any computable operation satisfying some mild conditions.
\end{abstract}

\section{Spectral Reals}

We assume familiarity with basic first-order logic and its model theory at the level of standard introductory texts such as Ebbinghaus, Flum, and Thomas [9] and Enderton [10]. Recall that the spectrum of a sentence $\phi$ in first-order logic is the set of nonnegative integers $n$ such that $\phi$ has a model of cardinality $n$.

In their survey [7], Durand et al. introduce a new class of real numbers induced by spectra.

Received May 19, 2011; accepted October 9, 2013

First published online March 24, 2016

2010 Mathematics Subject Classification: Primary 03C13, 68Q15, 68Q19; Secondary 11U05, $11 \cup 09$

Keywords: first-order logic, spectral reals, computability theory, computational complexity

C 2016 by University of Notre Dame $\quad 10.1215 / 00294527-3489987$ 


\title{
Some Remarks on Real Numbers Induced by First-Order Spectra
}

\author{
Sune Kristian Jakobsen and Jakob Grue Simonsen
}

\begin{abstract}
The spectrum of a first-order sentence is the set of natural numbers occurring as the cardinalities of finite models of the sentence. In a recent survey, Durand et al. introduce a new class of real numbers, the spectral reals, induced by spectra and pose two open problems associated to this class. In the present note, we answer these open problems as well as other open problems from an earlier, unpublished version of the survey.

Specifically, we prove that (i) every algebraic real is spectral, (ii) every automatic real is spectral, (iii) the subword density of a spectral real is either 0 or 1, and both may occur, and (iv) every right-computable real number between 0 and 1 occurs as the subword entropy of a spectral real.

In addition, Durand et al. note that the set of spectral reals is not closed under addition or multiplication. We extend this result by showing that the class of spectral reals is not closed under any computable operation satisfying some mild conditions.
\end{abstract}

\section{Spectral Reals}

We assume familiarity with basic first-order logic and its model theory at the level of standard introductory texts such as Ebbinghaus, Flum, and Thomas [9] and Enderton [10]. Recall that the spectrum of a sentence $\phi$ in first-order logic is the set of nonnegative integers $n$ such that $\phi$ has a model of cardinality $n$.

In their survey [7], Durand et al. introduce a new class of real numbers induced by spectra.

Received May 19, 2011; accepted October 9, 2013

First published online March 24, 2016

2010 Mathematics Subject Classification: Primary 03C13, 68Q15, 68Q19; Secondary 11U05, $11 \cup 09$

Keywords: first-order logic, spectral reals, computability theory, computational complexity

C 2016 by University of Notre Dame $\quad 10.1215 / 00294527-3489987$ 


\title{
Some Remarks on Real Numbers Induced by First-Order Spectra
}

\author{
Sune Kristian Jakobsen and Jakob Grue Simonsen
}

\begin{abstract}
The spectrum of a first-order sentence is the set of natural numbers occurring as the cardinalities of finite models of the sentence. In a recent survey, Durand et al. introduce a new class of real numbers, the spectral reals, induced by spectra and pose two open problems associated to this class. In the present note, we answer these open problems as well as other open problems from an earlier, unpublished version of the survey.

Specifically, we prove that (i) every algebraic real is spectral, (ii) every automatic real is spectral, (iii) the subword density of a spectral real is either 0 or 1, and both may occur, and (iv) every right-computable real number between 0 and 1 occurs as the subword entropy of a spectral real.

In addition, Durand et al. note that the set of spectral reals is not closed under addition or multiplication. We extend this result by showing that the class of spectral reals is not closed under any computable operation satisfying some mild conditions.
\end{abstract}

\section{Spectral Reals}

We assume familiarity with basic first-order logic and its model theory at the level of standard introductory texts such as Ebbinghaus, Flum, and Thomas [9] and Enderton [10]. Recall that the spectrum of a sentence $\phi$ in first-order logic is the set of nonnegative integers $n$ such that $\phi$ has a model of cardinality $n$.

In their survey [7], Durand et al. introduce a new class of real numbers induced by spectra.

Received May 19, 2011; accepted October 9, 2013

First published online March 24, 2016

2010 Mathematics Subject Classification: Primary 03C13, 68Q15, 68Q19; Secondary 11U05, $11 \cup 09$

Keywords: first-order logic, spectral reals, computability theory, computational complexity

C 2016 by University of Notre Dame $\quad 10.1215 / 00294527-3489987$ 


\title{
Some Remarks on Real Numbers Induced by First-Order Spectra
}

\author{
Sune Kristian Jakobsen and Jakob Grue Simonsen
}

\begin{abstract}
The spectrum of a first-order sentence is the set of natural numbers occurring as the cardinalities of finite models of the sentence. In a recent survey, Durand et al. introduce a new class of real numbers, the spectral reals, induced by spectra and pose two open problems associated to this class. In the present note, we answer these open problems as well as other open problems from an earlier, unpublished version of the survey.

Specifically, we prove that (i) every algebraic real is spectral, (ii) every automatic real is spectral, (iii) the subword density of a spectral real is either 0 or 1, and both may occur, and (iv) every right-computable real number between 0 and 1 occurs as the subword entropy of a spectral real.

In addition, Durand et al. note that the set of spectral reals is not closed under addition or multiplication. We extend this result by showing that the class of spectral reals is not closed under any computable operation satisfying some mild conditions.
\end{abstract}

\section{Spectral Reals}

We assume familiarity with basic first-order logic and its model theory at the level of standard introductory texts such as Ebbinghaus, Flum, and Thomas [9] and Enderton [10]. Recall that the spectrum of a sentence $\phi$ in first-order logic is the set of nonnegative integers $n$ such that $\phi$ has a model of cardinality $n$.

In their survey [7], Durand et al. introduce a new class of real numbers induced by spectra.

Received May 19, 2011; accepted October 9, 2013

First published online March 24, 2016

2010 Mathematics Subject Classification: Primary 03C13, 68Q15, 68Q19; Secondary 11U05, $11 \cup 09$

Keywords: first-order logic, spectral reals, computability theory, computational complexity

C 2016 by University of Notre Dame $\quad 10.1215 / 00294527-3489987$ 


\title{
Some Remarks on Real Numbers Induced by First-Order Spectra
}

\author{
Sune Kristian Jakobsen and Jakob Grue Simonsen
}

\begin{abstract}
The spectrum of a first-order sentence is the set of natural numbers occurring as the cardinalities of finite models of the sentence. In a recent survey, Durand et al. introduce a new class of real numbers, the spectral reals, induced by spectra and pose two open problems associated to this class. In the present note, we answer these open problems as well as other open problems from an earlier, unpublished version of the survey.

Specifically, we prove that (i) every algebraic real is spectral, (ii) every automatic real is spectral, (iii) the subword density of a spectral real is either 0 or 1, and both may occur, and (iv) every right-computable real number between 0 and 1 occurs as the subword entropy of a spectral real.

In addition, Durand et al. note that the set of spectral reals is not closed under addition or multiplication. We extend this result by showing that the class of spectral reals is not closed under any computable operation satisfying some mild conditions.
\end{abstract}

\section{Spectral Reals}

We assume familiarity with basic first-order logic and its model theory at the level of standard introductory texts such as Ebbinghaus, Flum, and Thomas [9] and Enderton [10]. Recall that the spectrum of a sentence $\phi$ in first-order logic is the set of nonnegative integers $n$ such that $\phi$ has a model of cardinality $n$.

In their survey [7], Durand et al. introduce a new class of real numbers induced by spectra.

Received May 19, 2011; accepted October 9, 2013

First published online March 24, 2016

2010 Mathematics Subject Classification: Primary 03C13, 68Q15, 68Q19; Secondary 11U05, $11 \cup 09$

Keywords: first-order logic, spectral reals, computability theory, computational complexity

C 2016 by University of Notre Dame $\quad 10.1215 / 00294527-3489987$ 


\title{
Some Remarks on Real Numbers Induced by First-Order Spectra
}

\author{
Sune Kristian Jakobsen and Jakob Grue Simonsen
}

\begin{abstract}
The spectrum of a first-order sentence is the set of natural numbers occurring as the cardinalities of finite models of the sentence. In a recent survey, Durand et al. introduce a new class of real numbers, the spectral reals, induced by spectra and pose two open problems associated to this class. In the present note, we answer these open problems as well as other open problems from an earlier, unpublished version of the survey.

Specifically, we prove that (i) every algebraic real is spectral, (ii) every automatic real is spectral, (iii) the subword density of a spectral real is either 0 or 1, and both may occur, and (iv) every right-computable real number between 0 and 1 occurs as the subword entropy of a spectral real.

In addition, Durand et al. note that the set of spectral reals is not closed under addition or multiplication. We extend this result by showing that the class of spectral reals is not closed under any computable operation satisfying some mild conditions.
\end{abstract}

\section{Spectral Reals}

We assume familiarity with basic first-order logic and its model theory at the level of standard introductory texts such as Ebbinghaus, Flum, and Thomas [9] and Enderton [10]. Recall that the spectrum of a sentence $\phi$ in first-order logic is the set of nonnegative integers $n$ such that $\phi$ has a model of cardinality $n$.

In their survey [7], Durand et al. introduce a new class of real numbers induced by spectra.

Received May 19, 2011; accepted October 9, 2013

First published online March 24, 2016

2010 Mathematics Subject Classification: Primary 03C13, 68Q15, 68Q19; Secondary 11U05, $11 \cup 09$

Keywords: first-order logic, spectral reals, computability theory, computational complexity

C 2016 by University of Notre Dame $\quad 10.1215 / 00294527-3489987$ 


\title{
Some Remarks on Real Numbers Induced by First-Order Spectra
}

\author{
Sune Kristian Jakobsen and Jakob Grue Simonsen
}

\begin{abstract}
The spectrum of a first-order sentence is the set of natural numbers occurring as the cardinalities of finite models of the sentence. In a recent survey, Durand et al. introduce a new class of real numbers, the spectral reals, induced by spectra and pose two open problems associated to this class. In the present note, we answer these open problems as well as other open problems from an earlier, unpublished version of the survey.

Specifically, we prove that (i) every algebraic real is spectral, (ii) every automatic real is spectral, (iii) the subword density of a spectral real is either 0 or 1, and both may occur, and (iv) every right-computable real number between 0 and 1 occurs as the subword entropy of a spectral real.

In addition, Durand et al. note that the set of spectral reals is not closed under addition or multiplication. We extend this result by showing that the class of spectral reals is not closed under any computable operation satisfying some mild conditions.
\end{abstract}

\section{Spectral Reals}

We assume familiarity with basic first-order logic and its model theory at the level of standard introductory texts such as Ebbinghaus, Flum, and Thomas [9] and Enderton [10]. Recall that the spectrum of a sentence $\phi$ in first-order logic is the set of nonnegative integers $n$ such that $\phi$ has a model of cardinality $n$.

In their survey [7], Durand et al. introduce a new class of real numbers induced by spectra.

Received May 19, 2011; accepted October 9, 2013

First published online March 24, 2016

2010 Mathematics Subject Classification: Primary 03C13, 68Q15, 68Q19; Secondary 11U05, $11 \cup 09$

Keywords: first-order logic, spectral reals, computability theory, computational complexity

C 2016 by University of Notre Dame $\quad 10.1215 / 00294527-3489987$ 


\title{
Some Remarks on Real Numbers Induced by First-Order Spectra
}

\author{
Sune Kristian Jakobsen and Jakob Grue Simonsen
}

\begin{abstract}
The spectrum of a first-order sentence is the set of natural numbers occurring as the cardinalities of finite models of the sentence. In a recent survey, Durand et al. introduce a new class of real numbers, the spectral reals, induced by spectra and pose two open problems associated to this class. In the present note, we answer these open problems as well as other open problems from an earlier, unpublished version of the survey.

Specifically, we prove that (i) every algebraic real is spectral, (ii) every automatic real is spectral, (iii) the subword density of a spectral real is either 0 or 1, and both may occur, and (iv) every right-computable real number between 0 and 1 occurs as the subword entropy of a spectral real.

In addition, Durand et al. note that the set of spectral reals is not closed under addition or multiplication. We extend this result by showing that the class of spectral reals is not closed under any computable operation satisfying some mild conditions.
\end{abstract}

\section{Spectral Reals}

We assume familiarity with basic first-order logic and its model theory at the level of standard introductory texts such as Ebbinghaus, Flum, and Thomas [9] and Enderton [10]. Recall that the spectrum of a sentence $\phi$ in first-order logic is the set of nonnegative integers $n$ such that $\phi$ has a model of cardinality $n$.

In their survey [7], Durand et al. introduce a new class of real numbers induced by spectra.

Received May 19, 2011; accepted October 9, 2013

First published online March 24, 2016

2010 Mathematics Subject Classification: Primary 03C13, 68Q15, 68Q19; Secondary 11U05, $11 \cup 09$

Keywords: first-order logic, spectral reals, computability theory, computational complexity

C 2016 by University of Notre Dame $\quad 10.1215 / 00294527-3489987$ 


\title{
Some Remarks on Real Numbers Induced by First-Order Spectra
}

\author{
Sune Kristian Jakobsen and Jakob Grue Simonsen
}

\begin{abstract}
The spectrum of a first-order sentence is the set of natural numbers occurring as the cardinalities of finite models of the sentence. In a recent survey, Durand et al. introduce a new class of real numbers, the spectral reals, induced by spectra and pose two open problems associated to this class. In the present note, we answer these open problems as well as other open problems from an earlier, unpublished version of the survey.

Specifically, we prove that (i) every algebraic real is spectral, (ii) every automatic real is spectral, (iii) the subword density of a spectral real is either 0 or 1, and both may occur, and (iv) every right-computable real number between 0 and 1 occurs as the subword entropy of a spectral real.

In addition, Durand et al. note that the set of spectral reals is not closed under addition or multiplication. We extend this result by showing that the class of spectral reals is not closed under any computable operation satisfying some mild conditions.
\end{abstract}

\section{Spectral Reals}

We assume familiarity with basic first-order logic and its model theory at the level of standard introductory texts such as Ebbinghaus, Flum, and Thomas [9] and Enderton [10]. Recall that the spectrum of a sentence $\phi$ in first-order logic is the set of nonnegative integers $n$ such that $\phi$ has a model of cardinality $n$.

In their survey [7], Durand et al. introduce a new class of real numbers induced by spectra.

Received May 19, 2011; accepted October 9, 2013

First published online March 24, 2016

2010 Mathematics Subject Classification: Primary 03C13, 68Q15, 68Q19; Secondary 11U05, $11 \cup 09$

Keywords: first-order logic, spectral reals, computability theory, computational complexity

C 2016 by University of Notre Dame $\quad 10.1215 / 00294527-3489987$ 


\title{
Some Remarks on Real Numbers Induced by First-Order Spectra
}

\author{
Sune Kristian Jakobsen and Jakob Grue Simonsen
}

\begin{abstract}
The spectrum of a first-order sentence is the set of natural numbers occurring as the cardinalities of finite models of the sentence. In a recent survey, Durand et al. introduce a new class of real numbers, the spectral reals, induced by spectra and pose two open problems associated to this class. In the present note, we answer these open problems as well as other open problems from an earlier, unpublished version of the survey.

Specifically, we prove that (i) every algebraic real is spectral, (ii) every automatic real is spectral, (iii) the subword density of a spectral real is either 0 or 1, and both may occur, and (iv) every right-computable real number between 0 and 1 occurs as the subword entropy of a spectral real.

In addition, Durand et al. note that the set of spectral reals is not closed under addition or multiplication. We extend this result by showing that the class of spectral reals is not closed under any computable operation satisfying some mild conditions.
\end{abstract}

\section{Spectral Reals}

We assume familiarity with basic first-order logic and its model theory at the level of standard introductory texts such as Ebbinghaus, Flum, and Thomas [9] and Enderton [10]. Recall that the spectrum of a sentence $\phi$ in first-order logic is the set of nonnegative integers $n$ such that $\phi$ has a model of cardinality $n$.

In their survey [7], Durand et al. introduce a new class of real numbers induced by spectra.

Received May 19, 2011; accepted October 9, 2013

First published online March 24, 2016

2010 Mathematics Subject Classification: Primary 03C13, 68Q15, 68Q19; Secondary 11U05, $11 \cup 09$

Keywords: first-order logic, spectral reals, computability theory, computational complexity

C 2016 by University of Notre Dame $\quad 10.1215 / 00294527-3489987$ 


\title{
Some Remarks on Real Numbers Induced by First-Order Spectra
}

\author{
Sune Kristian Jakobsen and Jakob Grue Simonsen
}

\begin{abstract}
The spectrum of a first-order sentence is the set of natural numbers occurring as the cardinalities of finite models of the sentence. In a recent survey, Durand et al. introduce a new class of real numbers, the spectral reals, induced by spectra and pose two open problems associated to this class. In the present note, we answer these open problems as well as other open problems from an earlier, unpublished version of the survey.

Specifically, we prove that (i) every algebraic real is spectral, (ii) every automatic real is spectral, (iii) the subword density of a spectral real is either 0 or 1, and both may occur, and (iv) every right-computable real number between 0 and 1 occurs as the subword entropy of a spectral real.

In addition, Durand et al. note that the set of spectral reals is not closed under addition or multiplication. We extend this result by showing that the class of spectral reals is not closed under any computable operation satisfying some mild conditions.
\end{abstract}

\section{Spectral Reals}

We assume familiarity with basic first-order logic and its model theory at the level of standard introductory texts such as Ebbinghaus, Flum, and Thomas [9] and Enderton [10]. Recall that the spectrum of a sentence $\phi$ in first-order logic is the set of nonnegative integers $n$ such that $\phi$ has a model of cardinality $n$.

In their survey [7], Durand et al. introduce a new class of real numbers induced by spectra.

Received May 19, 2011; accepted October 9, 2013

First published online March 24, 2016

2010 Mathematics Subject Classification: Primary 03C13, 68Q15, 68Q19; Secondary 11U05, $11 \cup 09$

Keywords: first-order logic, spectral reals, computability theory, computational complexity

C 2016 by University of Notre Dame $\quad 10.1215 / 00294527-3489987$ 


\title{
Some Remarks on Real Numbers Induced by First-Order Spectra
}

\author{
Sune Kristian Jakobsen and Jakob Grue Simonsen
}

\begin{abstract}
The spectrum of a first-order sentence is the set of natural numbers occurring as the cardinalities of finite models of the sentence. In a recent survey, Durand et al. introduce a new class of real numbers, the spectral reals, induced by spectra and pose two open problems associated to this class. In the present note, we answer these open problems as well as other open problems from an earlier, unpublished version of the survey.

Specifically, we prove that (i) every algebraic real is spectral, (ii) every automatic real is spectral, (iii) the subword density of a spectral real is either 0 or 1, and both may occur, and (iv) every right-computable real number between 0 and 1 occurs as the subword entropy of a spectral real.

In addition, Durand et al. note that the set of spectral reals is not closed under addition or multiplication. We extend this result by showing that the class of spectral reals is not closed under any computable operation satisfying some mild conditions.
\end{abstract}

\section{Spectral Reals}

We assume familiarity with basic first-order logic and its model theory at the level of standard introductory texts such as Ebbinghaus, Flum, and Thomas [9] and Enderton [10]. Recall that the spectrum of a sentence $\phi$ in first-order logic is the set of nonnegative integers $n$ such that $\phi$ has a model of cardinality $n$.

In their survey [7], Durand et al. introduce a new class of real numbers induced by spectra.

Received May 19, 2011; accepted October 9, 2013

First published online March 24, 2016

2010 Mathematics Subject Classification: Primary 03C13, 68Q15, 68Q19; Secondary 11U05, $11 \cup 09$

Keywords: first-order logic, spectral reals, computability theory, computational complexity

C 2016 by University of Notre Dame $\quad 10.1215 / 00294527-3489987$ 


\title{
Some Remarks on Real Numbers Induced by First-Order Spectra
}

\author{
Sune Kristian Jakobsen and Jakob Grue Simonsen
}

\begin{abstract}
The spectrum of a first-order sentence is the set of natural numbers occurring as the cardinalities of finite models of the sentence. In a recent survey, Durand et al. introduce a new class of real numbers, the spectral reals, induced by spectra and pose two open problems associated to this class. In the present note, we answer these open problems as well as other open problems from an earlier, unpublished version of the survey.

Specifically, we prove that (i) every algebraic real is spectral, (ii) every automatic real is spectral, (iii) the subword density of a spectral real is either 0 or 1, and both may occur, and (iv) every right-computable real number between 0 and 1 occurs as the subword entropy of a spectral real.

In addition, Durand et al. note that the set of spectral reals is not closed under addition or multiplication. We extend this result by showing that the class of spectral reals is not closed under any computable operation satisfying some mild conditions.
\end{abstract}

\section{Spectral Reals}

We assume familiarity with basic first-order logic and its model theory at the level of standard introductory texts such as Ebbinghaus, Flum, and Thomas [9] and Enderton [10]. Recall that the spectrum of a sentence $\phi$ in first-order logic is the set of nonnegative integers $n$ such that $\phi$ has a model of cardinality $n$.

In their survey [7], Durand et al. introduce a new class of real numbers induced by spectra.

Received May 19, 2011; accepted October 9, 2013

First published online March 24, 2016

2010 Mathematics Subject Classification: Primary 03C13, 68Q15, 68Q19; Secondary 11U05, $11 \cup 09$

Keywords: first-order logic, spectral reals, computability theory, computational complexity

C 2016 by University of Notre Dame $\quad 10.1215 / 00294527-3489987$ 


\title{
Some Remarks on Real Numbers Induced by First-Order Spectra
}

\author{
Sune Kristian Jakobsen and Jakob Grue Simonsen
}

\begin{abstract}
The spectrum of a first-order sentence is the set of natural numbers occurring as the cardinalities of finite models of the sentence. In a recent survey, Durand et al. introduce a new class of real numbers, the spectral reals, induced by spectra and pose two open problems associated to this class. In the present note, we answer these open problems as well as other open problems from an earlier, unpublished version of the survey.

Specifically, we prove that (i) every algebraic real is spectral, (ii) every automatic real is spectral, (iii) the subword density of a spectral real is either 0 or 1, and both may occur, and (iv) every right-computable real number between 0 and 1 occurs as the subword entropy of a spectral real.

In addition, Durand et al. note that the set of spectral reals is not closed under addition or multiplication. We extend this result by showing that the class of spectral reals is not closed under any computable operation satisfying some mild conditions.
\end{abstract}

\section{Spectral Reals}

We assume familiarity with basic first-order logic and its model theory at the level of standard introductory texts such as Ebbinghaus, Flum, and Thomas [9] and Enderton [10]. Recall that the spectrum of a sentence $\phi$ in first-order logic is the set of nonnegative integers $n$ such that $\phi$ has a model of cardinality $n$.

In their survey [7], Durand et al. introduce a new class of real numbers induced by spectra.

Received May 19, 2011; accepted October 9, 2013

First published online March 24, 2016

2010 Mathematics Subject Classification: Primary 03C13, 68Q15, 68Q19; Secondary 11U05, $11 \cup 09$

Keywords: first-order logic, spectral reals, computability theory, computational complexity

C 2016 by University of Notre Dame $\quad 10.1215 / 00294527-3489987$ 